\title{
Reducing patient-staff contact in fast-track total hip arthroplasty has no effect on patient-reported out- comes, but decreases satisfaction amongst patients with self-perceived complications: analysis of 211 patients
}

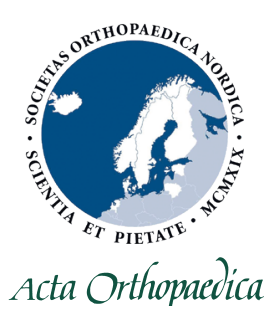

\author{
Jens B HANSEN ${ }^{1,2}$, Jens $\mathrm{F}$ L SØRENSEN ${ }^{1}$, Eva N GLASSOU ${ }^{3,4}$, Morten HOMILIUS ${ }^{3}$, \\ and Torben B HANSEN ${ }^{3,5}$
}

\begin{abstract}
${ }^{1}$ Department of Sociology, Environmental and Business Economics at the University of Southern Denmark, Esbjerg; ${ }^{2}$ NIDO | danmark, Gødstrup Hospital; ${ }^{3}$ University Clinic of Hand, Hip and Knee Surgery, Department of Orthopaedics, Gødstrup Hospital; ${ }^{4}$ Department of Quality, Gødstrup Hospital; ${ }^{5}$ Department of Clinical Medicine, Aarhus University, Denmark Correspondence: jensbh@sam.sdu.dk

Submitted 2021-10-01. Accepted 2021-12-17
\end{abstract}

Background and purpose - Several studies have compared fast-track with conventional pathways for total hip arthroplasty (THA) patients, but none have compared different fast-track pathways. Due to COVID-19 restrictions, our department had to minimize patient-staff contact in the THA pathway. First, telephone consultations were implemented instead of an outpatient clinic visit and subsequently preoperative patient education was discontinued. This enabled us to compare patient-reported outcomes and satisfaction among 3 fast-track pathways.

Patients and methods - We collected data from patients treated for hip osteoarthritis with THA at Gødstrup Hospital between 2018 and 2021. The patients had experienced 1 of 3 pathways and were interviewed via telephone between 2 and 6 months after discharge. We analyzed the influence of patient pathway on patient-reported pain and mobility level, self-perceived complications, and compliance using logistic regression. We then compared the pathway's effect on patient satisfaction both for the total sample and for the patients who experienced complications.

Results - The amount of patient-staff contact in the patient pathway did not have any influence on patientreported outcomes or the probability of self-perceived complications. For the full sample, patient-staff contact had no statistically significant influence on patient satisfaction either, but for the subgroup of patients experiencing complications, the pathways with less patient-staff contact reduced satisfaction. Patient satisfaction was primarily related to pain and mobility outcomes.
Interpretation - Our results indicate that reducing patient-staff contact in fast-track THA can be done without influencing mobility and pain outcomes, but the overall satisfaction among patients with self-perceived complications will be negatively affected.

Fast-track surgery has now become the preferred pathway for total hip arthroplasty (THA) in Denmark (1). Compared with conventional THA, it has shown to be cost-efficient by both reducing expenses for the hospital and shortening the length of stay for the patient $(2,3)$. After also displaying either neutral or positive effects on postoperative complications, readmission rates, or mortality $(2,4)$, the success criteria for fast-track THA gradually evolved towards a greater emphasis on patientreported outcomes (PROs) and satisfaction (5-7). Here too, the results have generally favored fast-track pathways $(\mathbf{8 , 9})$.

From this perspective, it is surprising that there have been no studies directly comparing the effect of different fast-track THA pathways seen from the patient's perspective. Although there have been qualitative studies into pathway optimization (10-13), their recommendations of enhancing preoperative patient education (PPE) and increasing patient-staff contact after discharge are inconsistent with the literature on conventional THA $(14,15)$.

We examined the effects of both pre- and postoperative patient-staff contact in fast-track THA. Due to COVID-19 restrictions on physical contact, our department had to reduce 
patient-staff contact on the fast-track THA pathway to a minimum. This was first done by applying telephone consultation as postoperative control, and subsequently by discontinuing PPE. As a consequence, we were able to compare 2 different pathways with a reduced amount of both pre- and postoperative patient-staff contact with already attained data on our initial fast-track pathway. The question hence became whether the decrease in patient-staff contact affected PROs and satisfaction.

\section{Patients and methods \\ Design}

This single-center study compared patient satisfaction and PROs of patients on 3 different fast-track THA pathways (P3; P2; P1), with different levels of both pre- and post-operative contact with the patient, executed over a period of $2 \frac{1}{2}$ years. Disregarding the initial meeting, where the indication for the operation is confirmed and the surgery is scheduled, our original pathway program (P3) contained 3 physical meetings: first, a PPE group meeting, where the patients were presented with the surgical procedure, informed on the process around it, and given their first instructions by the physiotherapist. After hospital discharge, there was then a follow-up consultation in the outpatient clinic after 2 weeks, where the sutures were removed, and, lastly, a concluding consultation in the outpatient clinic 6 weeks after discharge, amounting to 3 physical extra-operative meetings between patient and staff.

In P2, we implemented telephone consultation for the final 6-week consultation, which was estimated by the staff to reduce consultation time by one-third. In P1, we also discontinued the PPE meeting. The number of physical attendances at the clinic consequently decreased from 3, to 2, to 1: P3 had 1 PPE meeting and 2 postoperative physical consultations; P2 had 1 PPE meeting, 1 postoperative physical consultation, and 1 telephone consultation; $\mathrm{P} 1$ had 1 postoperative physical consultation and 1 telephone consultation. On all pathways, the patients were encouraged to contact the outpatient clinic should any questions regarding their recovery process arise, or if they experienced complications.

\section{Sample and data collection}

This study included 211 patients treated for hip osteoarthritis by primary cementless arthroplasty at Gødstrup Hospital interviewed 55-263 days after discharge. We used voluntary response sampling, which was stratified into 3 sub-populations divided between patients on the 3 different pathways. Patients of P3 $(n=64)$ were operated on between July and October 2018 and interviewed in December 2018. Patients of P2 $(n=71)$ were operated on between July and November 2020 and interviewed in February 2021. Patients of P1 ( $\mathrm{n}=$ 76) were operated on between December 2020 and April 2021 and interviewed in February and May 2021.
The interviews were done via telephone at a non-scheduled time. The patients had been advised beforehand via mail, where they were given instructions on how to decline participation. They were also given the opportunity to decline at the interview onset. Here, the interviewer also made it clear that he was not part of the clinical staff. 1 patient refused to participate, and we were unable to reach 28 patients, leaving us with a response rate of $88 \%$ of all 240 operated on in the given period.

We used a structured interview approach with fixed category questions constructed for subsequent quantitative analysis as specified below. The interviews had a mean length of 12 minutes (SD 5). Readmissions for hip-related issues, such as dislocation, infection, or thromboembolic complications, during the first 30 days were registered using data from the hospital database and validated through a review of patient records.

\section{Measurements}

\section{Dependent variables}

Our first set of dependent variables are pain level, mobility level, and patient satisfaction. The patients were asked to determine their overall satisfaction on a 5-point Likert scale ranging from "Very dissatisfied" to "Very satisfied." Pain and mobility scores were patient assessed matching the EQ-5D-3L 3-point scales, measuring pain as either "Extreme pain," "Moderate pain," or "No pain," and mobility as "Confined to bed," "Some problems walking," and "No problems walking." Due to a strong left-skewed distribution, these 3 variables were converted to binary variables denoting whether the patient reported being "Very satisfied," having "No pain," or "No problems walking."

We used a binary variable for 30-day readmissions and a binary variable to measure the patients' self-perceived postoperative complications, defined as whether or not the patient had had any non-prescheduled additional postoperative consultations regarding their recovery with either the outpatient clinic or their general practitioner. Lastly, we used a binary variable for compliance, understood as the patient reporting having adhered to the standard rehabilitative exercise program prescribed by the department.

\section{Independent variables}

Our main independent variable was a 3-point categorical variable denoting the 3 different patient pathways (P3; P2; P1). In all our adjusted models we used the following 5 variables per default: (i) sex (binary); (ii) age (numerical); (iii) distance to treatment (numerical) measured in kilometers from the patient's "nearest town sign" to the outpatient clinic as shown by Google Maps fastest route and back again; (iv) days since operation (numerical), showing the days between the surgery and the data collection; (v) compliance (binary).

\section{Statistics}

We first presented descriptive statistics for all single variables across the 3 pathways and compared the variables across path- 
Table 1. Sample characteristics, satisfaction rates, and PROs stratified by pathway. Values are $\mathrm{n}(\%)$ unless otherwise specified

\begin{tabular}{lccccc}
\hline Factor & $\begin{array}{c}\text { Total } \\
(\mathrm{n}=211)\end{array}$ & $\begin{array}{c}\text { Pathway 3 } \\
(\mathrm{n}=64)\end{array}$ & $\begin{array}{c}\text { Pathway } 2 \\
(\mathrm{n}=71)\end{array}$ & $\begin{array}{c}\text { Pathway 1 } \\
(\mathrm{n}=76)\end{array}$ & p-value a \\
\hline Age b & $65(10)$ & $68(7)$ & $63(11)$ & $64(10)$ & 0.005 \\
Male & $125(59)$ & $32(50)$ & $45(63)$ & $48(63)$ & 0.2 \\
Distance, km b & $66(37)$ & $72(38)$ & $70(39)$ & $59(32)$ & 0.06 \\
Days since operation b & $122(46)$ & $99(28)$ & $161(41)$ & $105(38)$ & $<0.001$ \\
Satisfaction & & & & & 0.5 \\
$\quad$ Very dissatisfied & $3(1)$ & $0(0)$ & $2(3)$ & $1(1)$ & \\
$\quad$ Dissatisfied & $8(4)$ & $2(3)$ & $1(1)$ & $5(7)$ & \\
Ambivalent & $13(6)$ & $2(3)$ & $6(8)$ & $5(7)$ & \\
$\quad$ Satisfied & $25(12)$ & $7(1)$ & $7(10)$ & $11(14)$ & \\
$\quad$ Very satisfied & $162(77)$ & $53(83)$ & $55(77)$ & $54(71)$ & \\
Pain & & & & & 0.9 \\
$\quad$ Extreme pain & $9(4)$ & $3(5)$ & $2(3)$ & $4(5)$ & \\
Moderate pain & $75(36)$ & $21(33)$ & $26(37)$ & $28(37)$ & \\
$\quad$ No pain & $127(60)$ & $40(63)$ & $43(61)$ & $44(58)$ & \\
Mobility & & & & & 0.8 \\
$\quad$ Confined to bed & $0(0)$ & $0(0)$ & $0(0)$ & $0(0)$ & \\
$\quad$ Some problems walking & $66(31)$ & $18(28)$ & $23(32)$ & $25(33)$ & \\
$\quad$ No problems walking & $145(69)$ & $46(72)$ & $48(68)$ & $51(67)$ & \\
Compliance (yes) & $132(63)$ & $47(73)$ & $41(58)$ & $44(58)$ & 0.09 \\
Readmitted (yes) & $0(0)$ & $0(0)$ & $0(0)$ & $0(0)$ & \\
Complications (yes) & $68(32)$ & $20(31)$ & $24(34)$ & $24(32)$ & 0.9 \\
\hline
\end{tabular}

a $p$-values compare the variables across the 3 pathways. For continuous variables, we use the ANOVA test, which tests the null hypothesis that the means in pathways 1,2, and 3 are of equal size. For categorical variables, we use the chi-square test, which tests the null hypothesis that the 2 categorical variables are independent of each other. $p$-values below 0.05 reject the null hypothesis.

b Values are mean (SD)

ways using bivariate tests (ANOVA or chi-square) (Table 1). Next, we proceeded to multivariate analyses, where we first analyzed the relationship between patient pathway and pain, mobility, complications, and compliance in both crude and adjusted logistic regression models (Table 2). Then we analyzed the determinants of responding "Very satisfied" through logistic regression, first in a crude model, then in 2 adjusted models containing respectively the default adjustments and additional adjustments for PROs and complications (Table 3). Lastly, we created regression models, which separated the patients who had complications from those who had not and analyzed the determinants for satisfaction in these 2 subsets in both crude and adjusted models (Table 4).

All the results were reported through odds ratios (ORs) and 95\% confidence intervals (CIs), hence p-values $<0.05$ were considered to be statistically significant. The data analysis was conducted using R.

\section{Ethics, funding, data sharing, and potential conflicts of interest}

Approval by the Regional Ethics Committee was not required (case number 1-10-72-181-20), as it was based on data from quality-control studies. The study did not receive any funding. Data can be made available on request to the corresponding author. None of the authors have any conflicts of interest to declare.

\section{Results}

The 211 patients were divided among the 3 pathways, with a slight decrease in number from P3 to P1 (Table 1). Patient characteristics were similar across pathways, although P3 had a statistically significantly higher mean age $(p=0.005), P 2$ had more days since operation $(p=<0.001)$, and $\mathrm{P} 1$ had less travel time, although only at borderline significance $(p=0.06)$. The distribution of satisfaction, PROs, and complications was shown to be considerably left skewed.

Table 2. Fast-track THA pathway's influence on PROs, complications, and compliance $(\mathrm{N}=211)$. Logistic regression with crude and adjusted models. Values are odds ratio $(95 \% \mathrm{Cl})$

\begin{tabular}{|c|c|c|c|c|c|}
\hline Factor & & No pain & No mobility problem & No complications & Compliance \\
\hline \multicolumn{6}{|l|}{ Crude model } \\
\hline Pathway 2 (ref. pathway 3) & 0.9 & $(0.5-1.8)$ & $0.8 \quad(0.4-1.7)$ & $0.9 \quad(0.4-1.8)$ & $0.5 \quad(0.2-1.02)$ \\
\hline Pathway 1 (ref. pathway 3 ) & 0.8 & $(0.4-1.6)$ & $0.8 \quad(0.4-1.6)$ & $0.98(0.5-2.0)$ & $0.5(0.2-1.02)$ \\
\hline Pseudo $R^{2}$ (Nagelkerke) & 0.00 & & 0.00 & 0.00 & 0.03 \\
\hline \multicolumn{6}{|l|}{ Adjused model } \\
\hline Pathway 2 (ref. pathway 3) & 1.0 & $(0.4-2.4)$ & $1.3(0.5-3.4)$ & $0.9 \quad(0.4-1.8)$ & $0.4(0.2-0.98)^{c}$ \\
\hline Pathway 1 (ref. pathway 3) & 0.8 & $(0.4-1.7)$ & $0.9(0.4-1.9)$ & $0.97(0.5-2.1)$ & $0.5 \quad(0.2-1.1)$ \\
\hline Male (ref. female) & 1.1 & $(0.6-1.9)$ & $1.2(0.7-2.3)$ & $1.7(0.9-3.0)$ & $0.8 \quad(0.4-1.4)$ \\
\hline Age $^{b}$ & 1.0 & $(0.99-1.05)$ & $1.0(0.99-1.06)$ & $1.0(0.99-1.05)$ & $0.99(0.97-1.03)$ \\
\hline Distance, $\mathrm{km}^{\mathbf{b}}$ & 0.99 & $9(0.98-1.00)$ & $0.99(0.98-1.00)$ & $1.0(0.99-1.01)$ & $1.0(0.99-1.01)$ \\
\hline Days since operation ${ }^{\mathbf{b}}$ & 1.0 & (0.99-1.01) & $0.99(0.99-1.00)$ & $0.99(0.99-1.01)$ & $1.0(0.99-1.01)$ \\
\hline Compliance (ref. no compl.) & 1.1 & $(0.6-2.0)$ & $2.0(1.1-3.8)^{a}$ & $0.7(0.4-1.3)$ & \\
\hline Pseudo $R^{2}$ (Nagelkerke) & 0.03 & & 0.08 & 0.05 & 0.05 \\
\hline
\end{tabular}


Table 3. Determinants of very high satisfaction $(N=211)$. Logistic regression with crude and 2 adjusted models. Values are odds ratio $(95 \% \mathrm{Cl})$

\begin{tabular}{lcccc}
\hline & \multicolumn{3}{c}{ Very high overall satisfaction } \\
& \multicolumn{1}{c}{ Crude } & Adjusted (1) & Adjusted (2) \\
\hline Pathway 2 (ref. pathway 3) & $0.7(0.3-1.7)$ & $1.0(0.3-3.0)$ & $0.95(0.2-4.1)$ \\
Pathway 1 (ref. pathway 3) & $0.5(0.2-1.2)$ & $0.5(0.2-1.2)$ & $0.5(0.2-1.5)$ \\
Male (ref. female) & & $2.1(1.1-4.2)$ & a & $2.3(0.9-6.0)$ \\
Age b & $1.0(0.99-1.07)$ & $1.0(0.98-1.08)$ \\
Distance, km b & $0.99(0.98-1.00)$ & $0.99(0.98-1.01)$ \\
Days since operation b & $0.99(0.99-1.01)$ & $0.99(0.99-1.01)$ \\
Compliance (ref. no compliance) & $1.9(0.96-3.8)$ & $2.5(0.93-7.0)$ \\
No mobility problems (ref. some problems walking) & $5.8(2.2-15.1)^{\text {a }}$ \\
No pain (ref. extreme or moderate pain) & & $10.1(3.5-29.7)^{\text {a }}$ \\
No complications (ref. some complications) & & $4.2(1.6-11.0)^{\text {a }}$ \\
Pseudo R ${ }^{2}$ (Nagelkerke) & 0.02 & 0.12 & 0.59
\end{tabular}

a $p$-value $<0.05$.

b Continuous variables without reference category.

Table 4. Determinants of very high satisfaction for 2 sub-samples. Logistic regression with crude and adjusted models. Values are odds ratio $(95 \% \mathrm{Cl})$

\begin{tabular}{|c|c|c|}
\hline Very high overall satisfaction & $\begin{array}{l}\text { Patients with } \\
\text { no self-perceived } \\
\text { postoperative } \\
\text { complications } \\
(n=143)\end{array}$ & $\begin{array}{l}\text { Patients with } \\
\text { some self-perceived } \\
\text { postoperative } \\
\text { complications } \\
(n=68)\end{array}$ \\
\hline \multicolumn{3}{|l|}{ Crude model } \\
\hline Pathway 2 (ref. pathway 3) & $2.0(0.6-7.5)$ & $0.3(0.06-0.97)^{a}$ \\
\hline Pathway 1 (ref. pathway 3) & $1.2(0.4-3.8)$ & $0.2(0.04-0.6)^{a}$ \\
\hline Pseudo R² (Nagelkerke) & 0.02 & 0.16 \\
\hline \multicolumn{3}{|l|}{ Adjusted model } \\
\hline Pathway 2 (ref. pathway 3) & $1.9(0.3-10.5)$ & $0.3(0.06-0.97)^{a}$ \\
\hline Pathway 1 (ref. pathway 3) & $1.4(0.4-5.3)$ & $0.2(0.04-0.8)^{a}$ \\
\hline Male (ref. female) & $2.8 \quad(0.95-8.1)$ & $1.1(0.4-3.4)$ \\
\hline Age $^{b}$ & $1.1 \quad(0.99-1.1)$ & $1.0 \quad(0.96-1.08)$ \\
\hline Distance, $\mathrm{km}^{\mathbf{b}}$ & $0.99(0.97-1.00)$ & $0.99(0.97-1.01)$ \\
\hline Days since operation ${ }^{\mathbf{b}}$ & $1.0(0.99-1.02)$ & $0.99(0.97-1.01)$ \\
\hline Compliance (ref. no compliance) & $3.6(1.2-10.6)^{a}$ & $2.6(0.7-9.3)$ \\
\hline Pseudo $R^{2}$ (Nagelkerke) & 0.17 & 0.24 \\
\hline
\end{tabular}

a $p$-value $<0.05$.

${ }^{b}$ Continuous variables without reference category.

ate regression did not show the pathways to affect the probability of having respectively no pain, no mobility problems, or no complications (Table 2). However, compliance with the rehabilitative exercise program doubled the likelihood of having "No problems" with mobility (OR 2; CI 1.1-3.8), and patients in $\mathrm{P} 2$ were less likely to comply with the recommended rehabilitation (OR 0.4; CI 0.2-0.98)). Notably, Nagelkerke's pseudo-R2 showed all models to explain very little of the variance in outcome, with the crude models explaining between $0 \%$ and $3 \%$.

\section{The effect of patient pathway, PROs, and complications on patient satisfac- tion}

There was no statistically significant bivariate correlation between pathway and satisfaction (Table 1). In multivariate regression, patient pathway was also unrelated to satisfaction in both the crude and the 2 adjusted regression models (Table 3 ). In the smaller adjusted model, being male was related to a higher probability of being very satisfied, but this relation disappeared when including pain, mobility, and complications in the larger model. These 3 variables, having no mobility problems (OR 5.8; CI 2.2-15), no pain (OR 10.1; CI 3.5-30), and no complications (OR 4.2; CI 1.6-11), appeared to be good predictors of patients reporting "Very high" satisfaction. A pseudo-R2 score of 0.6 also indicated that including variables for mobility, pain, and complication heightened the explanatory power of the model markedly.

Dividing the sample into 2 subsets with 1 group having had complications, and 1 group having not, showed pathway design

But where the distribution of complications appeared constant across pathways, we noticed a slight, but statistically insignificant, proportional drop in patients reporting "No pain" ( $p$ $=0.9)$ and "No problems walking" $(\mathrm{p}=0.8)$ from P3 to P1. A similar, albeit larger, proportional drop from P3 to P1 was found in patient satisfaction $(\mathrm{p}=0.5)$. There were no 30-day readmissions (Table 1).

\section{Pathway's effect on PROs, complications, and com- pliance}

Patient pathway did not have an effect on patient-reported pain and mobility outcome, or on the probability of selfperceived complications. The bivariate analysis showed no statistically significant variation in PROs, complications, or compliance between pathways (Table 1), and the multivari- to be unrelated to being fully satisfied for patients with no complications, but P1 (OR 0.2; CI 0.04-0.6) and P2 (OR 0.3; $0.06-0.97)$ to be negatively correlated in the crude model for patients with complications (Table 4). In the adjusted model, P1 still affected the satisfaction rate for patients with complications negatively (OR 0.2; CI 0.04-0.8), while P2 showed no statistically significant effect.

\section{Discussion}

We found no relation between pathway design and either patient-reported pain or mobility outcome, or the likelihood of self-perceived postoperative complications. While qualitative studies have argued that more focus on the period after dis- 
charge may positively influence pain management, recovery, and functional outcome, our results indicate that this may not be the case for pain and mobility outcomes $(11,12)$. Our rates for both outcomes were comparable to corresponding Swedish numbers on all pathways, albeit slightly higher (8). Thus, although qualitative studies have recommended PPE before fast-track THA to improve PROs, we did not find evidence to support this recommendation $(13,16)$. Similarly, we did not find longer lasting postoperative face-to-face consultation to be superior to telephone consultation regarding PROs. Our results are congruent with the literature on conventional THA, where the benefits of PPE have been shown to be unsure, and the effects of pre- and postoperative exercise interventions on recovery appear trivial at best $(14,15,17)$.

In the full sample, we found no statistically significant relation between patient pathway and satisfaction, with all 3 pathways showing satisfaction rates that matched the general tendency for THA (18). We did, however, find a statistically insignificant decrease in the satisfaction rate as patient-staff contact decreased, but in our small sample we cannot identify an association. Likewise, since we, in congruence with the literature (19), found the main factors behind patient satisfaction after THA to be pain and mobility outcome, as well as not experiencing complications, the high success rate for these outcomes may conceal a negative trend in the satisfaction with care that did not manifest itself in the patients' overall evaluations.

Nevertheless, the drop in satisfaction in the full sample appeared to be mainly driven by a change in satisfaction rate among the subgroup of patients experiencing self-perceived complications. When we distinguished between patients with and without complications, we saw that converting to telephone consultation may have had a negative influence on the satisfaction of patients with complications, although the results were conflicted. Furthermore, discontinuing PPE was shown unambiguously to reduce satisfaction for those patients with complications. As the pain and mobility outcomes were unaffected by these changes in patient-staff contact, the reasons for these negative effects are unclear. We propose 2 explanations.

First, the lower satisfaction rate amongst the patients experiencing complications on the pathway with the least patientstaff contact (P1) may have been caused by these patients missing the psychological benefits of PPE. Since one main purpose of PPE is anxiety reduction, it is possible that those patients who had not undergone PPE were less tolerant towards complications (17). But given that we measured complications as self-perceived, and the prevalence of patients experiencing such complications is constant across pathways, we are hesitant to support this hypothesis.

Second, an alternate explanation for the lower satisfaction rate could be hindsight bias in the evaluations. People generally struggle to process random outcomes, especially regarding negative life events, and the patients on the pathways with the lowest amount of patient-staff contact would have been more likely to counterfactually attribute their self-perceived complications to insufficient support from the healthcare personnel in the recovery process $(\mathbf{2 0}, 21)$. For fast-track THA programs to better comply with patients who experience complications, further investigations into similar secondary mechanisms behind patient satisfaction are warranted.

At first glance, reducing patient-staff contact in the fasttrack THA thus appears to be a trade-off between, on the one hand, the health personnel saving time they can hence spend on other tasks, and, on the other hand, a slight reduction in the overall satisfaction rate springing from the patients with complications. We must, however, take into account too that patient-staff consultations are also an expense in time and effort for the patient. Because our study could not confirm that more consultations had an effect on PROs or the probability of complications, it could be considered an expense imposed on the patients, which had a questionable return for the majority of them.

Our results thus indicate that minimizing patient-staff contact by discontinuing PPE and utilizing telephone consultation can be done safely, and therefore can be considered for permanent implementation beyond COVID-19 restrictions. This spares time and effort for both the health personnel and the majority of patients, at the cost of lowering the satisfaction rate amongst the minority of patients with complications.

Using a single-center design is a strength of our study. It meant that the results should not be affected by confounders related to differences in staff or institution, since the same surgeons and nursing staff were present across pathways. It also meant that the patients came from the same geographical area, holding demographic and cultural variations nearly constant. Furthermore, the use of telephone interviews was shown to produce a high response rate, benefiting the validity by reducing the otherwise considerable participation bias found in questionnaire-based designs (22). It also enabled the interviewer to actively control for a potential COVID-19 related acceptance of limitations on patient-staff contact by patients, by asking the interviewees to disregard any COVID19 considerations in their evaluation.

There are several limitations to our study. It is a clear limitation that this was a small sample retrospective design. Retrospective studies may suffer from recall bias, but as we were able to adjust for days since operation, and found the early evaluations not to differ from the later evaluations, we had no immediate reason to suspect recall bias in our data. Adjusting for days since operation without it showing an impact on PROs, satisfaction, or the probability of complications also means that the potentially problematic unequal distribution of days since operation across pathways should not be considered a major weakness.

Regrettably, we were not able to adjust for possible prognostic factors such as BMI and comorbidity. This is a considerable limitation, even though the results for including BMI are 
conflicting, and, while there is a moderate level of evidence for comorbidity as a predictor for recovery in conventional THA, research solely on fast-track primary cementless THA points toward no relation between ASA score and postoperative pain and functional level (23-25).

Furthermore, measuring the patients' self-perceived complications as non-prescheduled additional consultations with either the outpatient clinic or GP had both pros and cons. Due to the rarity of readmissions after THA, our small sample size was not able to register any occurrences of 30-day readmissions relating to clinical complications such as infection, luxation, or a thromboembolic event on any of the pathways. A larger sample is hence needed to confirm this non-effect. Instead, the use of self-perceived complications as measured by the probability of additional consultations enabled us to register variations in the probability of complications as seen from the patient's perspective, while also giving us the crucial ability to detect whether a decrease in the number of prescheduled consultations simply resulted in an increased number of non-prescheduled consultations. In this way, we could assess whether reducing patient-staff contact actually did reduce patient-staff contact.

In conclusion, we found no influence between patient pathway and patient-reported pain and mobility outcome, after comparing 3 different fast-track THA pathways with different amounts of patient-staff contact. Similarly, we found no statistically significant relationship between the different pathways and patient satisfaction on a general level, but the pathway with the least contact had lower satisfaction rates amongst the patients with self-perceived complications. Thus, our results suggest that orthopedic departments can safely decrease patient-staff contact in fast-track THA programs, but might experience a minor negative effect on patient satisfaction as a result.

Author statement in accordance with CRediT (Contributor Roles Taxonomy): JH: conceptualization (lead), methodology, formal analysis (lead), investigation, data-curation (lead), writing - original draft, writing - review and editing (lead), visualization, project administration. JS: validation, formal analysis (supporting), data curation (supporting), writing - review and editing (supporting), supervision. TH: conceptualization (supporting), resources, writing - review and editing (supporting). EG: writing - review and editing (supporting), supervision. MH: conceptualization (supporting).

The authors wish to thank all the patients involved for their responsiveness in interviews.

Acta thanks Urban Berg for help with peer review of this study.

1. Hansen T B. Fast track in hip arthroplasty. EFORT Open Rev 2017; 2(5): 179-88. doi: 10.1302/2058-5241.2.160060.

2. Zhu S, Qian W, Jiang $\mathbf{C}$, Ye $\mathbf{C}$, Chen $\mathbf{X}$. Enhanced recovery after surgery for hip and knee arthroplasty: a systematic review and meta-analysis. Postgrad Med J 2017; 93(1106): 736-42. doi: 10.1136/postgradmedj-2017-134991.
3. Andreasen S E, Holm H B, Jørgensen M, Gromov K, KjærsgaardAndersen P, Husted H. Time-driven activity-based cost of fast-track total hip and knee arthroplasty. J Arthroplasty 2017; 32(6): 1747-55. doi: 10.1016/j.arth.2016.12.040.

4. Deng Q F, Gu H Y, Peng W Y, Zhang Q, Huang Z D, Zhang C, et al. Impact of enhanced recovery after surgery on postoperative recovery after joint arthroplasty: results from a systematic review and metaanalysis. Postgrad Med J 2018; 94(1118): 678-93. doi: 10.1136/postgradmedj-2018-136166.

5. Zywiel M G, Mahomed A, Gandhi R, Perruccio A V, Mahomed N N. Measuring expectations in orthopaedic surgery: a systematic review. Clin Orthop Relat Res 2013; 471(11): 3446-56. doi: 10.1007/s11999-0133013-8.

6. Graham B, Green A, James M, Katz J, Swiontkowski M. Measuring patient satisfaction in orthopaedic surgery. J Bone Joint Surg Am 2015; 97(1): 80-4. doi: 10.2106/JBJS.N.00811.

7. Strickland L H, Kelly L, Hamilton T W, Murray D W, Pandit H G, Jenkinson C. Early recovery following lower limb arthroplasty: qualitative interviews with patients undergoing elective hip and knee replacement surgery. Initial phase in the development of a patient-reported outcome measure. J Clin Nurs 2018; 27(13-14): 2598-608. doi: 10.1111/ jocn. 14086 .

8. Berg U, W-Dahl A, Rolfson O, Nauclér E, Sundberg M, Nilsdotter A. Influence of fast-track programs on patient-reported outcomes in total hip and knee replacement (THR/TKR) at Swedish hospitals 2011-2015: an observational study including 51,169 THR and 8,393 TKR operations. Acta Orthop 2020; 91(3): 306-12. doi: 10.1080/17453674.2020.1733375.

9. Winther S B, Foss O A, Wik T S, Davis S P, Engdal M, Jessen V, et al. 1-year follow-up of 920 hip and knee arthroplasty patients after implementing fast-track: good outcomes in a Norwegian university hospital. Acta Orthop 2015; 86(1): 78-85. doi: 10.3109/17453674.2014.957089.

10. Sjøveian A K H, Leegaard M. Hip and knee arthroplasty: patients' experiences of pain and rehabilitation after discharge from hospital. Int $\mathbf{J}$ Orthop Trauma Nurs 2017; 27: 28-35. doi: 10.1016/j.ijotn.2017.07.001.

11. Specht K, Agerskov H, Kjaersgaard-Andersen P, Jester R, Pedersen B D. Patients' experiences during the first 12 weeks after discharge in fast-track hip and knee arthroplasty: a qualitative study. Int J Orthop Trauma Nurs 2018; 31: 13-19. doi: 10.1016/j.ijotn.2018.08.002.

12. Berg U, Berg M, Rolfson O, Erichsen-Andersson A. Fast-track program of elective joint replacement in hip and knee: patients' experiences of the clinical pathway and care process. J Orthop Surg Res 2019; 14(1): 186. doi: 10.1186/s13018-019-1232-8.

13. Jansson M M, Harjumaa M, Puhto A P, Pikkarainen M. Patients' satisfaction and experiences during elective primary fast-track total hip and knee arthroplasty journey: a qualitative study. J Clin Nurs 2020; 29(3-4): 567-82. doi: 10.1111/jocn.15121.

14. Saueressig T, Owen P J, Zebisch J, Herbst M, Belavy D L. Evaluation of exercise interventions and outcomes after hip arthroplasty: a systematic review and meta-analysis. JAMA Netw Open 2021; 4(2): e210254. doi: 10.1001/jamanetworkopen.2021.0254.

15. Wang L, Lee M, Zhang Z, Moodie J, Cheng D, Martin J. Does preoperative rehabilitation for patients planning to undergo joint replacement surgery improve outcomes? A systematic review and meta-analysis of randomised controlled trials. BMJ Open 2016; 6(2): e009857. doi: 10.1136/bmjopen-2015-009857.

16. Specht K, Kjaersgaard-Andersen P, Pedersen B D. Patient experience in fast-track hip and knee arthroplasty: a qualitative study. J Clin Nurs 2016; 25(5-6): 836-45. doi: 10.1111/jocn.13121.

17. McDonald S, Page M J, Beringer K, Wasiak J, Sprowson A. Preoperative education for hip or knee replacement. Cochrane Database Syst Rev 2014; 2014(5): CD003526. doi: 10.1002/14651858.CD003526.pub3.

18. Kahlenberg C A, Nwachukwu B U, Schairer W W, Steinhaus M E, Cross M B. Patient satisfaction reporting after total hip arthroplasty: a systematic review. Orthopedics 2017; 40(3): e400-4. doi: 10.3928/ 01477447-20170120-04. 
19. Swarup I, Henn C M, Gulotta L V, Henn R F. Patient expectations and satisfaction in orthopaedic surgery: a review of the literature. J Clin Orthop Trauma 2019; 10(4): 755-60. doi: 10.1016/j.jcot.2018.08.008.

20. Kimhi S, Zysberg L. How people understand their world: perceived randomness of rare life events. J Psychol 2009; 143(5): 521-32. doi: 10.3200/JRL.143.5.521-532.

21. Roese N J, Vohs K D. Hindsight bias. Perspect Psychol Sci 2012; 7(5): 411-26. doi: 10.1177/17456916124543033.

22. Cheung K L, Ten Klooster P M, Smit C, De Vries H, Pieterse M E. The impact of non-response bias due to sampling in public health studies: a comparison of voluntary versus mandatory recruitment in a Dutch national survey on adolescent health. BMC Public Health 2017; 17(1): 276. doi: 10.1186/s12889-017-4189-8.
23. Leiss F, Götz J S, Maderbacher G, Meyer M, Reinhard J, Zeman F, et al. Excellent functional outcome and quality of life after primary cementless total hip arthroplasty (THA) using an enhanced recovery setup. J Clin Med 2021; 10(4): 621. doi: 10.3390/jcm10040621.

24. Elings J, Hoogeboom TJ, Van Der Sluis G, Van Meeteren N. What preoperative patient-related factors predict inpatient recovery of physical functioning and length of stay after total hip arthroplasty? A systematic review. Clin Rehabil 2015; 29(5): 477-92. doi: $10.1177 / 0269215514545349$

25. Hewlett-Smith N, Pope R, Furness J, Simas V, Hing W. Prognostic factors for inpatient functional recovery following total hip and knee arthroplasty: a systematic review. Acta Orthop 2020; 91(3): 313-318. doi: 10.1080/17453674.2020.1744852. 